\title{
Antibodies by Non-Febrile, Smear-Negative Individuals from a Malaria Epidemic-Prone Setting in Ethiopia are Strongly Reactive to Plasmodium falciparum Blood-Stage Vaccine Candidate Antigens
}

Hassen Mamo ${ }^{1,4 *}$, Nnaemeka C Iriemenam ${ }^{2-4}$, Klavs Berzins ${ }^{4}$ and Beyene Petros ${ }^{1}$

${ }^{1}$ Microbial, Cellular and Molecular Biology Department, College of Natural Sciences, Addis Ababa University, P. O. Box 1176, Addis Ababa, Ethiopia

${ }^{2}$ Division of Parasitic Diseases and Malaria, Center for Global Health, Centers for Disease Control and Prevention, Atlanta, Georgia, United States of America

${ }^{3}$ Department of Medical Microbiology and Parasitology, College of Medicine of the University of Lagos, Idi-araba, Lagos, Nigeria

${ }^{4}$ Department of Molecular Biosciences, The Wenner-Gren Institute, Department of Immunology, Stockholm University, Sweden

\begin{abstract}
Background: Plasmodium falciparum malaria remains a major public health concern globally though there is some decline due to scale-up of control efforts. Evaluation of the anti-malarial immune profile, in populations residing in epidemic-prone areas in the dry season or at the time when vector control largely reduced man-mosquito contact, would help predict malaria burden when future epidemics occur.
\end{abstract}

Methods: A cross-sectional study was designed to investigate antibody responses to four $P$. falciparum blood stage vaccine candidate antigens in non-febrile individuals from Shewa Robit in north-central Ethiopia where malaria transmission was expected at a minimal level as a result of the sampling season and effective vector control. Blood samples were analyzed microscopically for Plasmodium detection. The enzyme-linked immunosorbent assay (ELISA) was used to measure immunoglobulin (IgG) antibodies to apical membrane antigen 1 (AMA1), glutamaterich protein (GLURP) R2 region and merozoite surface protein 2 (MSP2) allelic variants.

Results: Study participants were smear-negative for Plasmodium infection. While $51(22 \%)$ of the participants reported that they had never been exposed to clinical malaria in life, 177 (78\%) reported at least one clinical malaria episode with laboratory confirmed $P$. falciparum infection. The antigens tested were well-recognized by the test sera although significant differences were observed in antibody prevalence and level between the different antigens and there was inter-individual variability. IgG response to the antigens showed age-related pattern but without evidence of relation with status and frequency of reported past exposure to clinical malaria.

Conclusion: The data suggests that individuals in an epidemic-prone malaria setting have reactive and stable antibodies that readily recognize $P$. falciparum blood-stage vaccine candidate antigens in the absence of slidepositivity. Analysis of age-related pattern in antibody level showed positive association with age but unrelated with increasing frequency of reported episode suggesting the role of intrinsic age-related factors in malaria immune maturation.

Keywords: Plasmodium falciparum; Malaria; Immunoglobulin; ELISA; Ethiopia; Vaccine; Antigen

Abbreviations: ALP: Alkaline Phosphatase; AMA: Apical Membrane Antigen; BSA: Bovine Serum Albumin; CI: Confidence Interval; ELISA: Enzyme-Linked Immunosorbent Assay; GLURP: Glutamate-Rich Protein; Ig: Immunoglobulin; ITN: InsecticideTreated Net; MSP: Merozoite Surface Protein; OD: Optical Density; PBS: Phosphate Buffered Saline; RDT: Rapid Diagnostic Test; SD: Standard Deviation; USA: United States of America

\section{Background}

Plasmodium falciparum malaria is among the leading causes of morbidity and mortality worldwide [1]. A widely held model of malaria immunity is slow age-related immune buildup [2]. It has also been argued that protective immunity to severe pathology is achieved relatively rapidly [3]. A work in South East Asia reported the development of malaria immunity as a function of age but independent of cumulative exposure [4]. Though it has been difficult to look into the effect of age on malaria immunity independent of exposure, these investigators argued that they managed to do so. Also, there was no significant difference in malaria antibody levels between individuals more extensively exposed and those with less exposure [5]. Another unresolved issue in malaria immunity is that antibody responses decline rapidly with time in the absence of persistent boost infections [6]. On the other hand, there are evidences contrary to this assertion.
It was reported that MSP2-specific antibodies in naturally exposed individuals in seasonal $P$. falciparum transmission areas remained remarkably stable from year to year [7].

This shows that there are unresolved issues regarding malaria immunity in natural settings demanding further research. If antibodies dissolve rapidly following parasite clearance sustained transmission reduction via vector control may pose the general population, in endemic areas, to greater risk of malaria-related morbidity and mortality when outbreaks occur. Thus evaluating antibody responses in the dry season would help evaluate the possible influence of vector control on malaria antibody stability.

Though malaria is a primary health concern, little data exist

${ }^{*}$ Corresponding author: Hassen Mamo, Microbial, Department of Molecular Biosciences, The Wenner-Gren Institute, Department of Immunology, Stockholm University, Sweden, E-mail: binmamo@yahoo.com

Received July 24, 2013; Accepted August 27, 2013; Published August 20, 2013 Citation: Mamo H, Iriemenam NC, Berzins K, Petros B (2013) Antibodies by Non-Febrile, Smear-Negative Individuals from a Malaria Epidemic-Prone Setting in Ethiopia are Strongly Reactive to Plasmodium falciparum Blood-Stage Vaccine Candidate Antigens. J Vaccines Vaccin 4: 200. doi: 10.4172/2157-7560.1000200

Copyright: ๑ $2013 \mathrm{Mamo} \mathrm{H}$, et al. This is an open-access article distributed under the terms of the Creative Commons Attribution License, which permits unrestricted use, distribution, and reproduction in any medium, provided the original author and source are credited. 
regarding malaria immunity in individuals living in endemic regions of Ethiopia particularly pertaining to the age-related pattern and duration of malaria immunity. In this regard the country is lagging behind many malaria endemic African countries some of which have already embarked on vaccine efficacy trials. Thus, the objective of this study was to evaluate the level of reactivity and stability of antibody responses to $P$. falciparum blood-stage antigens in asymptomatic individuals in a seasonal and unstable malaria locality in Ethiopia where vector control efforts effectively reduced transmission.

\section{Methods}

\section{Study site}

The climate of most Ethiopian malaria endemic regions is Sahelian with small rains ('Belg') in March and April and heavy rain (Kiremt) from June to September followed by a long, dry season from October to February. May is largely a dry month. Malaria in most parts of the country is meso-/hypo endemic and seasonal although stable and perennial transmission occurs in some western lowland and river basin areas. $P$. falciparum is the major species (60-70\%) in Ethiopia accounting for most malaria-related mortality and morbidity followed by $P$. vivax (30-40\%) [8].

This study was undertaken in Shewa Robit in north-central Ethiopia. Shewa Robit which is a District town is located in Amhara Regional State, Semiene Shewa Zone, Kewet District about $225 \mathrm{~km}$ to the north of Addis Ababa. The climate of Shewa Robit located at $1000^{\prime} 36^{\prime \prime} \mathrm{N}$ latitude and $39^{\circ} 54^{\prime} 10.8^{\prime \prime} \mathrm{E}$ longitude at 1380 masl is warm with $16.5^{\circ} \mathrm{C}$ mean annual minimum and $31^{\circ} \mathrm{C}$ maximum temperatures. The mean annual rainfall is $1007 \mathrm{~mm}$ [9]. While Shewa Robit town had a total population size of 17,575 (male 8874, female 8,701) Kewet District was populated by 118,333 (male 60,911; female 57,422) people [10]. The same report showed that the majority of the people in the district belonged to the Amhara ethnic group.

The town receives high rainfall during the main rainy season and is characterized by markedly unstable and seasonal malaria transmission standing among the major epidemics-prone areas in the country. Malaria control efforts were effectively in place in accordance with the national malaria control policy. The town had one healthcare center. There were health posts in all Kebeles (basic administrative units of a District) of the District to treat suspected malaria cases on the basis of rapid diagnostic test (RDT) and clinical diagnosis. Insecticide-treated net (ITN) distribution was $100 \%$ at least at household level according to health facility information system. The most recent epidemic in the area was in 2005 as the local healthcare system record showed.

\section{Ethical Considerations}

Prior to sampling ethical approval was obtained from the Biology Department Ethics Review Committee, Addis Ababa University and the Ethiopian Federal Ministry of Science and Technology. Informed consenting individuals (parents/legal guardians for children) participated in the study.

\section{Study design, sampling and infection detection}

A cross-sectional survey took place in 2008 in the dry season when malaria transmission was much declined. Consenting participants were requested to self-report their past malaria episode(s), if any, pointing out the number and most recent episode (year) including the Plasmodium species(s). Nearly all participant claims, for those who were treated within the local health center, were confirmed by cross-checking in the health record system. Five $\mathrm{ml}$ of venous blood was collected from each participant. Thick and thin blood-smears were prepared and microscopically screened for Plasmodium infection and species identification. Sera were separated and stored at $-20^{\circ} \mathrm{C}$ until analysis.

\section{Antibody detection}

ELISA: $P$. falciparum antigens AMA1 (3D7 strain) [11], MSP2 allelic families (3D7, FC27) [12] and GLURP R2 region (amino acids 705-1178) [13] were used for IgG analysis.

Positive high-titer reference serum from Sudanese donor with confirmed $P$. falciparum infection and negative control sera from malaria naïve Europeans were used for assay standardization and cutoff determination. The test protocol was following that of Iriemenam et al. [14]. Briefly, 96-well flat-bottomed ELISA plates (Costar, Cambridge, MA, USA) were coated using $100 \mu \mathrm{l} /$ well of solutions of AMA1, MSP23D7, MSP2-FC27 or GLURP-R2. The coating concentrations were $1 \mu \mathrm{g} / \mathrm{ml}$ for the first three antigens and $0.5 \mu \mathrm{g} / \mathrm{ml}$ for the last two in a coating buffer (phosphate buffered saline (PBS). The plates were incubated overnight at $4^{\circ} \mathrm{C}$. Using $100 \mu \mathrm{l} /$ well blocking buffer (sodium carbonate buffer ( $\mathrm{pH} 9.6$ ), containing $0.5 \%$ bovine serum albumin (BSA) the plates were blocked for $2 \mathrm{~h}$ at $37^{\circ} \mathrm{C}$. The plates were washed four times in a washing buffer [PBS with $0.1 \%$ Tween-20 and $0.15 \%$ Kathon (Mabtech, Nacka, Sweden)] in micro plate washer (Skan washer 300, CA, USA). Sera diluted 1:1000 in incubation buffer (PBS with $0.5 \%$ BSA) were loaded in duplicates and incubated for $1 \mathrm{~h}$ at $37^{\circ} \mathrm{C}$. To detect bound IgG antibodies, alkaline phosphatase (ALP) conjugated to goat anti-human IgG (1:2000) (Mabtech) was added and further incubated for $1 \mathrm{~h}$ at $37^{\circ} \mathrm{C}$. Finally, the assay was developed by adding $p$-nitrophenyl phosphate (Sigma-Aldrich $\mathrm{GmbH}$, Steinheim, Germany) in enzyme substrate buffer and keeping the plates in the dark. After 20 minutes the optical densities (OD) were read (at $405 \mathrm{~nm}$ ) using Molecular Devices (VmaxTM Kinetic micro plate reader, Menlo Park, USA). Samples having a discrepancy of $>25 \%$ between duplicates were retested. Background was determined from the OD values of blank wells and that was deducted from the mean OD reading of each sample. The cutoff point was set at the mean plus 3 standard deviations (SD) of negative-control sera.

\section{Data analysis}

First the normality and correctness of the data was checked and found that mean antibody levels were not normally distributed. Accordingly, nonparametric tests were used for all statistical analysis using SPSS version 17.0 for Windows (SPSS Inc., Chicago, IL, USA). Differences in antibody positivity between antigens, age groups as well as between reportedly exposed/unexposed groups to clinical malaria were assessed using the Fisher's exact test. Correlations between ODs for the different antigens and with donor age were determined using Pearson correlation. Pair-wise comparisons of the median ODs between different test groups were done using Mann-Whitney U (or Wilcoxon rank sum) test. The Kruskal-Wallis test was used to test for differences in antibody levels against more than two different groups. Two-sided $p<0.05,95 \%$ confidence intervals (CI) were considered significant.

\section{Results}

\section{Study participants and questionnaire data}

Two hundred twenty eight serum samples were collected from mostly adult consenting participants (age range 5-80 years, mean 28). All participants were non-febrile (axillary temperature $<37.5^{\circ} \mathrm{C}$ ) and smear-negative for Plasmodium infection during sampling. Fifty one 
Citation: Mamo H, Iriemenam NC, Berzins K, Petros B (2013) Antibodies by Non-Febrile, Smear-Negative Individuals from a Malaria Epidemic-Prone Setting in Ethiopia are Strongly Reactive to Plasmodium Falciparum Blood-Stage Vaccine Candidate Antigens. J Vaccines Vaccin 4: 200. doi: $10.4172 / 2157-7560.1000200$

Page 3 of 8

(22\%) of the study participants reported that they had not been exposed to any clinical malaria in life time in spite of residing in endemic area(s) (Table 1). The rest 177 (78\%) had at least one clinical malaria episode with laboratory confirmed $P$. falciparum infection. The subgroups among the exposed were those who had experienced episode(s) once (71/177(40.1\%) and more than once (106/177(59.9\%).

\section{Antibody positivity and level}

The antigens tested were well-recognized by the test sera MSP2FC27 being the leading and the GLURP-R2 reactive antibodies being relatively the least frequently detected (Table 2 ). There was no serum sample that was not IgG positive at least against one antigen. Thus $100 \%$ of the sera were positive for one or more antigens. However, some individuals were positive for one antigen and not for the other. Particularly, a large number (51\%) of the same sera that were positive for GLURP-R0 in a previous report [15] were not so for GLURP-R2. Similarly, a substantial number of individuals (about 20\%) were positive for MSP2-FC27 but not for MSP2-3D7. Conversely, few sera were positive for MSP2-3D7 or GLURP-R2 but not MSP2-FC27 or GLURP-R0, respectively.

The differences in IgG positivity between all antigens were significant except the comparison between AMA1 and MSP2-3D7. While anti-AMA1 antibody level was not significantly different from the level against MSP2-3D7 ( $p=0.072)$ and GLURP-R2 $(P=0.091)$ it was significantly lower than that to MSP2-FC27 $(p<0.0001)$. GLURP-R2 level was significantly lower than that to MSP2-3D7 $(p=0.010)$ and MSP2-FC27 $(p<0.0001)$. Anti-MSP2-FC27 IgG level was significantly higher than that to MSP2-3D7 $(p<0.0001)$.

Antibody levels to AMA1, MSP2-3D7, MSP2-FC27 and GLURP-R2 were significantly positively correlated with each other. Particularly, anti-MSP2-3D7 and MSP2-FC27, the two allelic variants of MSP2, levels have shown the strongest correlation $(p<0.0001, \mathrm{R}=0.651)$ (Figure 1). However, anti-GLURP-R0 (previous data from reference number [15]) and GLURP-R2 IgG levels were not significantly correlated.

\begin{tabular}{|c|c|c|c|}
\hline Sex & Male n (\%) & Female $\mathrm{n}(\%)$ & Total n (\%) \\
\hline \multicolumn{4}{|l|}{ Age (year) } \\
\hline $5-14$ & $7(3.0)$ & $13(5.7)$ & $20(8.7)$ \\
\hline$>14$ & $181(79.4)$ & $27(11.8)$ & 208(91.2) \\
\hline \multicolumn{4}{|l|}{ PME } \\
\hline Unexposed & $44(19.3)$ & $7(3.0)$ & $51(22.4)$ \\
\hline Exposed & $145(63.6)$ & & $32(14.0)$ \\
\hline \multicolumn{4}{|l|}{ LME } \\
\hline 1998-2004 & $28(12.2)$ & $5(2.2)$ & $33(14.4)$ \\
\hline 2005 & $36(15.8)$ & $9(3.9)$ & $45(19.7)$ \\
\hline 2006 & $17(7.4)$ & $1(0.40)$ & $18(7.8)$ \\
\hline 2007 & $30(13.2)$ & $8(3.5)$ & $38(16.7)$ \\
\hline 2008 & $34(14.9)$ & $9(3.9)$ & $43(18.8)$ \\
\hline
\end{tabular}

PME: Past Malaria Episode; LME: Latest Malaria Episode

Table 1: Demographic and questionnaire data for study participants.

\begin{tabular}{|c|c|c|}
\hline Antigen & Positivity rate $(\mathbf{\%})^{\mathbf{1}}(\mathbf{n}=\mathbf{2 2 8})$ & ${ }^{2}$ Median OD(IQR) \\
\hline AMA1 & $148(64.9)$ & $0.122(0.06)$ \\
\hline MSP2-3D7 & $150(65.8)$ & $0.121(0.02)$ \\
\hline MSP2-FC27 & $186(81.6)$ & $0.198(0.07)$ \\
\hline GLURP-R2 & $96(42.1)$ & $0.110(0.08)$ \\
\hline
\end{tabular}

Table 2: Antibody positivity rate and level to $P$. falciparum blood-stage vaccine candidate antigens in sera samples from individuals.

\section{Antibody response and self-reported past malaria episode}

IgG positivity to the different antigens differed between those who reported past malaria episode and those not (Table 3). While to AMA1 the unexposed group had significantly higher positivity rate than the exposed ( $p=0.008)$; for MSP2-3D7 $(p<0.0001)$, MSP2-FC27 $(p<0.0001)$ and GLURP-R2 ( $p=0.037)$ the opposite was noticed.

The difference in IgG positivity rate to the antigens between those who reported malaria episode once and more than once was less marked. However, the rate to AMA1 $(p=0.001)$ (higher in once) and MSP2-FC27 $(p<0.0001)$ (higher in more than once) indicated significance between the two groups. Anti-AMA1 positivity rate was the highest among those who reported their most recent episode in 19912004 (Table 4). Similarly, anti-AMA1 antibody level was significantly higher in individuals who reported that they were unexposed to malaria episode in life time than the exposed $(p<0.0001)$. On the other hand, the highest median antibody level against this antigen was for individuals whose latest reported episode was in 2008, the year of sample collection. Antibody level was also significantly higher in the group that reported exposure to malaria episode than those not exposed for MSP2-3D7 and MSP2-FC27. IgG levels to these antigens were significantly higher in individuals who reportedly had episodes more than once compared to those who had only one episode.

Antibody reactivity against all the four antigens significantly increased with increasing age though the degree of association was variable (Figures 2a-2d).

\section{Discussion}

Compared to other antigens tested the lowest anti-GLURP-R2 IgG prevalence and level was detected in the present study. A high IgG positivity rate was recorded in a holoendemic area from Liberia against the C-terminal 783 amino acids of the 220-kD GLURP (containing both R1 (489-705) and R2 (705-1179) regions) [16]. In a seasonal malaria transmission locality in the Gambia, IgG positivity rate reached $95 \%$ for this antigen [17]. Compared to IgG response against GLURP-R0 in our previous report in the same population [15] as well as other study in a different setting [18] the anti-GLURP-R2 response was lower.

The observed inconsistency may be explained in different ways. The

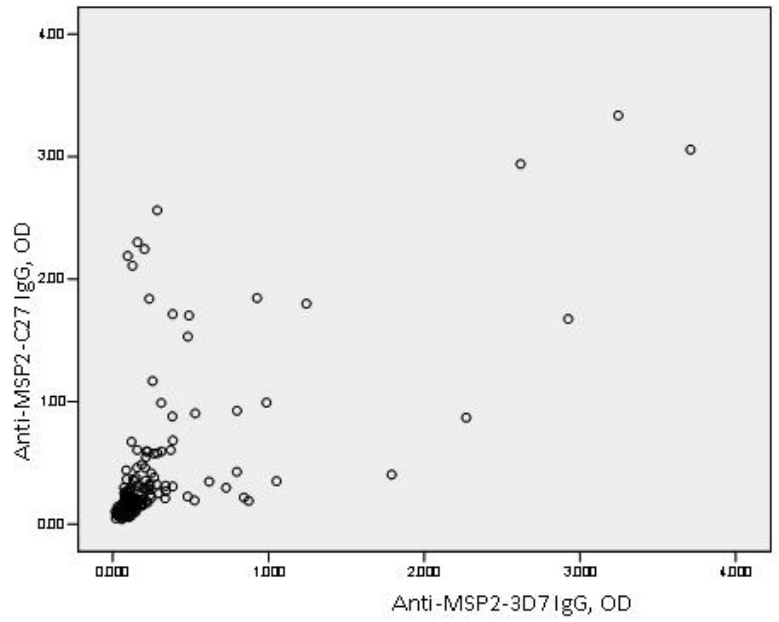

Figure 1: Correlation between IgG levels to MSP2-3D7 and MSP2FC27 $(r=0.651, p<0.0001)$. 
Citation: Mamo H, Iriemenam NC, Berzins K, Petros B (2013) Antibodies by Non-Febrile, Smear-Negative Individuals from a Malaria Epidemic-Prone Setting in Ethiopia are Strongly Reactive to Plasmodium Falciparum Blood-Stage Vaccine Candidate Antigens. J Vaccines Vaccin 4: 200. doi: $10.4172 / 2157-7560.1000200$

Page 4 of 8

\begin{tabular}{|l|l|l|l|l|l|l|l|l|l|}
\hline \multicolumn{1}{|c|}{ Status } & \multirow{2}{*}{$\mathbf{n}$} & \multicolumn{3}{c|}{ AMA1 } & \multicolumn{2}{c|}{ MSP2-3D7 } & \multicolumn{2}{c|}{ MSP2-FC27 } & \multicolumn{2}{c|}{ GLURP-R2 } \\
\cline { 3 - 11 } & & positivity no (\%) & \multicolumn{1}{c|}{ level } & positivity no (\%) & level & positivity no (\%) & level & positivity no (\%) & level \\
\hline Unexposed & 51 & $41(80.3)$ & $0.128(0.19)$ & $27(52.9)$ & $0.103(0.04)$ & $33(64.7)$ & $0.124(0.05)$ & $15(29.4)$ & $0.103(0.07)$ \\
\hline Exposed & 177 & $107(60.4)$ & $0.119(0.04)$ & $123(69.4)$ & $0.130(0.35)$ & $153(86.7)$ & $0.215(0.09)$ & $81(45.7)$ & $0.113(0.05)$ \\
\hline Single & 71 & $35(49.2)$ & $0.089(0.09)$ & $48(67.6)$ & $0.129(0.06)$ & $58(81.6)$ & $0.192(0.05)$ & $32(45.0)$ & $0.113(0.03)$ \\
\hline Multiple & 106 & $72(67.9)$ & $0.126(0.06)$ & $75(70.7)$ & $0.131(0.05)$ & $95(89.6)$ & $0.267(0.08)$ & $49(46.2)$ & $0.112(0.07)$ \\
\hline
\end{tabular}

Table 3: P. falciparum-specific IgG positivity and level (Median OD (IQR) and reported past malaria episodes.

\begin{tabular}{|c|c|c|c|c|c|c|c|c|c|}
\hline \multirow{2}{*}{ Status } & \multirow{2}{*}{$n$} & \multicolumn{2}{|c|}{ AMA1 } & \multicolumn{2}{|c|}{ MSP2-3D7 } & \multicolumn{2}{|c|}{ MSP2-FC27 } & \multicolumn{2}{|c|}{ GLURP-R2 } \\
\hline & & positivity no (\%) & level & positivity no (\%) & level & positivity no (\%) & level & positivity no (\%) & level \\
\hline 1991-2004 & 33 & $23(69.6)$ & $0.129(0.05)$ & $25(75.7)$ & $0.129(0.03)$ & $30(90.9)$ & $0.228(0.10)$ & $17(51.5)$ & $0.136(0.04)$ \\
\hline 2005 & 45 & $25(55.5)$ & $0.106(0.03)$ & $31(68.8)$ & $0.109(0.07)$ & 41 (91.1) & $0.200(0.06)$ & $18(40.0)$ & $0.097(0.07)$ \\
\hline 2006 & 18 & $10(55.5)$ & $0.099(0.08)$ & $14(77.7)$ & $0.145(0.05)$ & $17(94.4)$ & $0.238(0.12)$ & $10(55.5)$ & $0.140(0.05)$ \\
\hline 2007 & 38 & $22(57.8)$ & $0.107(0.06)$ & $21(55.2)$ & $0.138(0.08)$ & 29 (76.3) & $0.199(0.08)$ & $13(34.2)$ & $0.087(0.07)$ \\
\hline 2008 & 43 & 27 (62.7) & $0.130(0.44)$ & $32(74.4)$ & $0.134(0.04)$ & 36 (83.7) & $0.222(0.09)$ & $23(53.4)$ & $0.137(0.07)$ \\
\hline
\end{tabular}

Table 4: IgG positivity and level (median OD (IQR) to $P$. falciparum antigens and reported year of most recent malaria episode.

R0 region has limited variation between isolates from diverse geographic areas in contrast to the $\mathrm{R} 2$ repeat region which has been shown to have considerable polymorphism [19]. Therefore, the R2 region strain may not be common in the present study population or the strain-specific antibody induced may be short-lived, and was at undetectable level at the time of sample collection. Thus we suggest that compared to antiGLURP-R2 response antibodies induced to GLURP-R0 may be more stable, the protein is more antigenic or this particular region of GLURP is more prevalent in the studied population.

The lower anti-AMA1 IgG prevalence recorded in the present study (64.9\%) indicates apparent disparity from findings of other studies. A very high IgG positivity rate (96-100\%) was observed to AMA1 in other studies $[20,21]$. The apparent disparity between such reports and the present study may be attributable to differences in transmission level. Both Chelimo et al. [20] and Thomas et al. [21] reported from holoendemic areas but the present study was from a hypo endemic setting. However, equally high antibody level to AMA1 was detected from a seasonal transmission area [22] showing that endemicity level is a less likely explanation for the observed variability. On the contrary, the prevalence of $\operatorname{IgG}$ response to AMA1 was substantially lower (37\%), compared to the result in this study, in a low transmission setting in the Thailand [23]. Thus, reports of various studies on anti-AMA1 antibody response are less consistent. Apart from some differences in study design, several other factors are potential sources of variation. Among these; level of endemicity, time/season of antibody measurement, presence/absence of active infection and its duration and the level of parasitaemia, time of recent infection, or persistence of sub patent or sub-microscopic infections may make it difficult to compare results of different studies. More importantly antigen preparation or the exact constructs used can be source of differences as AMA1 is a polymorphic protein with great strain variations [24]. Furthermore, differences in the binding specificity and sensitivity of some ELISA regents, overall test system, host genetics, etc. may account for the apparent disparity.

Detection of high anti-MSP2 IgG positivity rate (up to $81.6 \%$ ) and level in this study is in agreement with reports of other studies. In an adult population in a seasonal transmission area in the Gambia, the anti-MSP2 antibody prevalence was very high, reaching 95\% [25]. Specifically, $81 \%$ of the individuals tested were positive for 3D7 and $86 \%$ were for serogroup FC27 being consistent with the present study, though the value for 3D7 was lower in the current study. More than $90 \%$ prevalence to the full-length proteins of both 3D7 and FC27 of MSP2 was observed in studies in Solomon Islands [26] and Papua New Guinea [27]. Additionally, anti-MSP2-FC27 IgG level was significantly higher than anti-MSP2-3D7 in Sudan [14] lending support to the present study. Some other studies detected IgG in all individuals to MSP2 in general [28] and to MSP2-3D7 specifically [22]. But another study measured only $15 \%$ IgG positivity rate to MSP2, lower than AMA1 [24]. The two allelic variants of MSP2 are widely distributed in general [29] but no specific information is available as to the relative distribution of these two serogroups in Ethiopia. The current finding, the first of its kind in the country, confirmed the occurrence of both allelic variants but probably in varying relative abundance at least in the studied area.

Antigen nature and level of exposure to various parasite strains may account for the inter-individual differences observed in the study. Inter-individual variability in malaria-specific antibody response in other malaria endemic areas was documented [30]. As a substantial number of individuals were positive for MSP2-FC27, but not MSP23D7; and similarly, $51 \%$ of sera positive for GLURP-R0 previously [15] were not so for GLURP-R2 currently suggest allele-specific antibody reaction. The induction of allele-specific natural antibodies to various $P$. falciparum recombinant antigens was similarly revealed in other studies [31,32]. The absence of significant correlation between anti-GLURP-R0 and GLURP-R2 antibody levels observed in the present study provides additional support in this regard. Detection of significant correlation between antibody levels to the allelic variants of MSP2 in the study goes in agreement with other reports [33]. This observation may be explained by exposure to both allelic families locally. Contrarily, Taylor et al. [25] found weak correlations between antibody responses to the two allelic variants of MSP2.

Recording significant correlation between anti-AMA1 and MSP2 total IgG responses in the present study agrees with other studies [34]. In general, correlation of antibodies to blood-stage antigens in individual serum suggests cross-reactivity between the antigens apart from the effect of exposure to multiple parasite strains and allelic forms. Such extensive inter-correlations between responses to a panel of vaccine candidate antigens in the current study have important implications for vaccine design efforts.

In the absence of slide-positivity and minimal transmission high seropositivity was noted indicating the stability of malarial antibody without high level infections. There are other evidences that suggested the long-lived nature of malaria antibodies in the absence of microscopically detectable infections $[23,35]$. Although the present study was cross-sectional in its design, information gathered through interview produced substantial amount of previous history of the 
Citation: Mamo H, Iriemenam NC, Berzins K, Petros B (2013) Antibodies by Non-Febrile, Smear-Negative Individuals from a Malaria Epidemic-Prone Setting in Ethiopia are Strongly Reactive to Plasmodium Falciparum Blood-Stage Vaccine Candidate Antigens. J Vaccines Vaccin 4: 200. doi: $10.4172 / 2157-7560.1000200$

Page 5 of 8

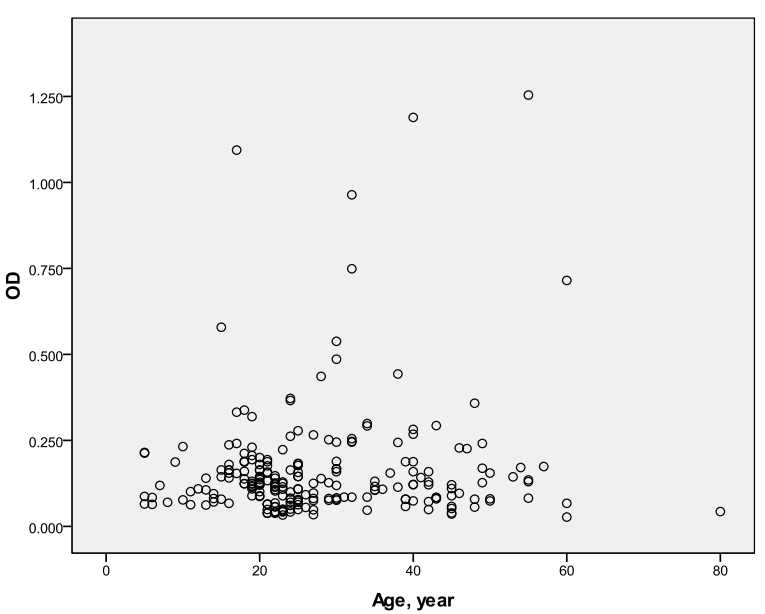

Figure 2a: Correlation between IgG level to AMA1 and age $(r=0.106$, $p=0.041)$.

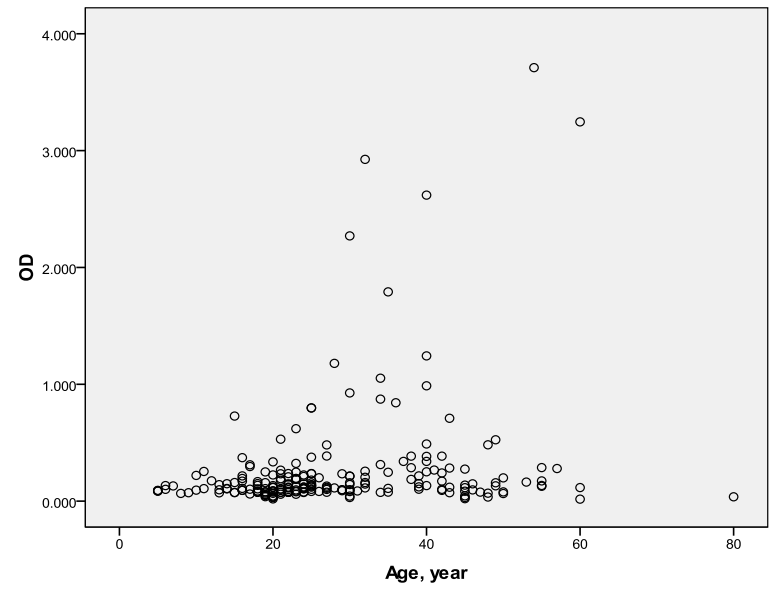

Figure 2b: Correlation between IgG level to MSP2-3D7 and age $(r=0.243, p<0.000)$.

participants by the assistance of local health facility record system that maintained malaria cases by passive case detection.

Anti-AMA1 positivity and level were significantly higher among individuals who reported no malaria episode in life than those who had. Further, IgG positivity rate for this antigen was greater in those who had clinical episode once than more than once. The same antiAMA1 IgG positivity rate was the highest among those who reported their most recent malaria episode in 1991-2004. But the highest median antibody level was for individuals whose latest episode was in 2008, the year of sample collection. These findings taken together suggest that protective AMA1-specific malaria antibody remains stable at detectable level for longer period but the level declines over time, though not significantly. Similarly, Giha et al. [36] observed significantly lower total IgG and subclass levels to Pf332-C231 in individuals exposed to clinical malaria at least once compared to those unexposed. Although in Ethiopia no information is yet available concerning the prevalence and distribution of the commonly used (3D7) and other strains of AMA1 the relatively recently exposed individuals (from 2005-2007) may have been exposed to other strains and produced antibodies that were non-cross-reacting or weakly cross-reacting with 3D7 (the strain used in the study). Conversely, the study participants might have been exposed to highly cross-reactive strains and remained asymptomatic during the study period though.

Antibodies to MSP2-3D7, MSP2-FC27 and GLURP-R2 may need more boosting to be maintained as individuals who reported clinical attacks had relatively higher and more stable responses in our study. Alternatively, the reportedly exposed having higher overall response to the two allelic variants of MSP2 may suggest that antibodies to these antigens are less protective. However, in a seasonal and unstable setting in the Gambia it was shown that antibodies to MSP2 did not significantly fluctuate between transmission seasons in protected adults [3].

Individuals who reported no past malaria episode but were seropositive in the study might have been asymptomatically infected and because of their higher response might have controlled parasitaemia. Similar findings where documented in Brazil [18] Sudan [36] and both in hypoendemic unstable transmission setting like the present one.

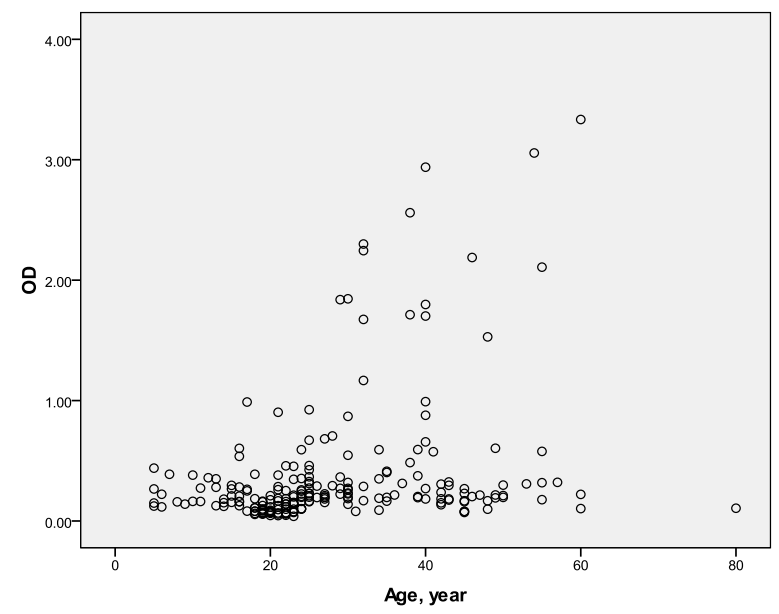

Figure 2c: Correlation between lgG level to MSP2-FC27 and age $(r=0.320, p<0.000)$

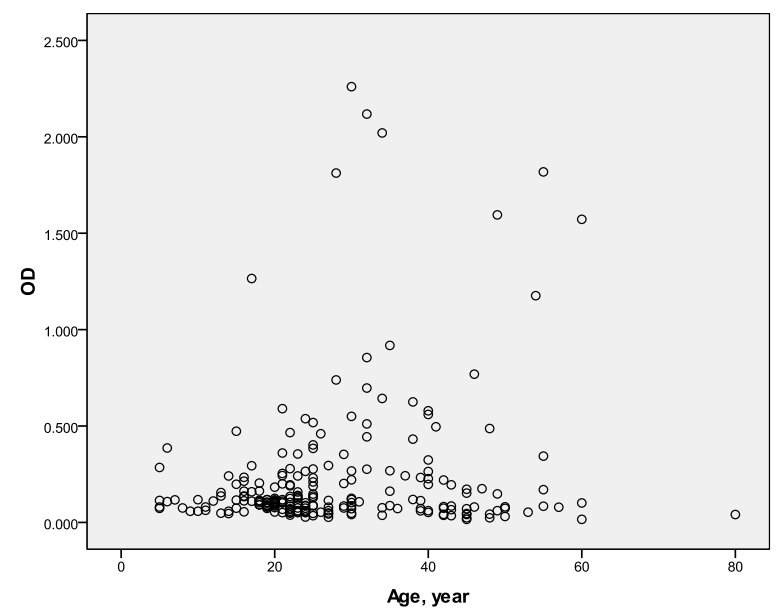

Figure 2d: Correlation between IgG level to GLURP-R2 and age $(\mathrm{r}=0.189, p=0.004)$. 
The persistence of chronic low-grade asymptomatic infections with $P$. falciparum among individuals in hypo endemic and unstable malaria transmission has been reported [33]. Since most of the study population have been permanently or for a long period of time residing in malaria endemic areas, their chance of remaining uninfected was very unlikely. Thus, some individuals might have induced and maintained protective antibody and thereby were protected from secondary infections. Or individuals in the group might have been sick sometime in the past but failed to remember and report their episodes. In addition, there is some limitation regarding the reliability of self-reported information. Some individuals may fail to recall the number and date of clinical episodes they experienced. However, it is likely that residents in endemic areas acquire malaria immunity somehow in the past following their first and second infections and then could report less episodes in the more recent years or may not even be exposed to clinical attack at all.

Also, they might have been misdiagnosed (false-negative for $P$. falciparum and were categorized among $P$. vivax positives). Alternatively, they might have been genuinely $P$. falciparum-unexposed but were $P$. vivax positive and because of cross-reaction they became $P$. falciparum seropositive. This is likely that a significant number of study populations reporting prior infection only with $P$. vivax were seropositive for $P$. falciparum antigens [23,37]. Thus, undiagnosed prior infection with $P$. falciparum or cross-reactivity of antibodies to the two species in the past may explain why participants who reported no episodes were seropositive. It might be just due to lower parasitaemia and could not induce clinical disease.

Whatsoever the explanation might be, detecting reactive antibody responses at a higher dilution (1:1000) in this group suggests that a significant proportion of study participants had malaria-specific antibodies that readily recognize blood-stage antigens of $P$. falciparum in Ethiopia in the absence of detectable parasitaemia by microscopy. Further, detection of high and comparable antibody positivity among individuals who reported being exposed only once and among those who had multiple episodes further suggests either sub-microscopic level of persistent infection capable of boosting the immune response, or simply the long-lived nature of antibody responses in the absence of persistent infections or re-infection for some antigens. The possibility of acquiring strong antibody levels following primary infection was also reported [38].

The age-related pattern of antibody level to the different antigens was also observed in other studies [34]. According to Tongren et al. [39] increasing age (and therefore malaria exposure) leads to increasing anti-MSP2 antibody prevalence and levels in more exposed individuals than less exposed ones. Apart from age; the duration and intensity of the infection, previous experience with the parasite strain, human genetics, nutritional status, other infections, etc may complicate the pattern of malaria immunity.

In the current study no significant difference between those who reported multiple past self-reported episodes and who had only a primary attack suggesting that previous experience (or cumulative exposure) is less influential at least in the study population. The duration of infection may be less important in antibody induction and maintenance as most individuals tested had access to treatment schemes which were made available through extensive network of treatment posts in addition to regular campaigns of control efforts that reduce man-mosquito contact and thus lower multiple infections and make parasitaemia less. Vector control reduces the force of infection and has implications for malaria immunity [40]. Further, the study area is characterized by markedly unstable seasonal transmission. All these factors are supposed to significantly reduce the duration as well as intensity of infection. Participants were slide-negative and had no clinical signs suggestive of malaria. Further, most had their most recent episodes three years back in 2005, the year in which the last epidemic occurred in the area. In spite of all these conditions that are perceived to delay the acquisition and persistence of immune response, the study evidenced significant antibody reactivity in most sera to most antigens tested. The relatively lower OD values observed however fit into the overall picture of low malaria transmission in the study area.

High IgG level was measured for relatively protected Liberian adults and primarily infected Swedes patients demonstrating that primary infection can result in antibody levels comparable to that seen among residents of endemic areas [41]. This is in line with the finding of the present study that people who reported malaria episode only once had higher responses suggesting the protective role of the antibodies. Further, when sera from individuals who experienced more extensive exposure and those with lesser exposure to $P$. falciparum were analyzed, no significant antibody titers were observed between the two groups [5]. A study which revealed the development of agedependent acquired malaria immunity within a two-year period of exposure in hyper endemic setting [42] corroborated this finding. Similarly, antibody responses to MSP1 showed no significant difference between individuals living in areas of low and high transmission of seasonal malaria [43]. Additionally, age-dependent, but only after brief exposure, development of malaria immunity was observed in a transmigrant population, originated from malaria free area, in a hyper endemic area [4]. In another study anti-MSP1 antibody was age- but not exposure-dependent for development [44].

It was suggested that some intrinsic age-related factors influence the development, and maintenance malaria immunity $[45,46]$. Similarly, the age-related pattern of antibody response in the present study may not reflect the effect of cumulative exposure to the parasite and as such possible gradual maturation of the immune system. It rather suggests the role of other intrinsic age-related factors in immune maturation.

\section{Conclusions}

A substantial number of individuals in the study area had antibodies that recognize $P$. falciparum blood stage vaccine antigens, MSP2-FC27 being the most frequently recognized. The extensive correlation between IgG levels against different blood stage antigens is an indication of cross-reactions and/or exposure of the study populations to different strains of $P$. falciparum. The documentation of antibody reactivity in slide-negative individuals with little history of self-reported malaria episode implies that malaria antibodies can be maintained detectable and in low transmission localities where seasonality as well as scale up of control effort significantly reduced transmission. This is not withstanding the potential importance of lowgrade asymptomatic submicroscopic infections in antibody duration. The study gives some clue on the impact of transmission reduction, via vector control, on the maintenance of protective malaria immunity.

\section{Competing Interests}

The authors declare that they have no competing interests.

\section{Authors' Contributions}

HM has conceived and designed the study; acquired, analysed and interpreted the data; drafted and revised the manuscript. NCI assisted with antibody measurement, interpreted the data and revised the manuscript; KB supervised laboratory work, interpreted the data and revised the manuscript; BP supervised fieldwork, interpreted the 
Citation: Mamo H, Iriemenam NC, Berzins K, Petros B (2013) Antibodies by Non-Febrile, Smear-Negative Individuals from a Malaria Epidemic-Prone Setting in Ethiopia are Strongly Reactive to Plasmodium Falciparum Blood-Stage Vaccine Candidate Antigens. J Vaccines Vaccin 4: 200. doi: $10.4172 / 2157-7560.1000200$

Page 7 of 8

data and revised the manuscript. All authors read and approved the submission of the manuscript for publication.

\section{Acknowledgement}

The Office of Vice President for Research and Publication and Dean of School of Graduate Studies, Addis Ababa University has provided a financial support for the study. Personal expense of the first author was covered by Ministry of Education, Federal Democratic Republic of Ethiopia during immunological data generation in Sweden. We are grateful to Prof Robin Anders, La Trobe University, Australia and Dr. Michael Theisen, Statens Serum Institute, Copenhagen, Denmark for kindly donating of $P$. falciparum AMA1 and MSP2, and GLURP antigens respectively. We thank Dr. Alemayehu Worku from School of Public Health, Addis Ababa University for statistical assistance. Government representatives, community leaders, health personnel, study populations and all other who collaborated in the study are duly acknowledged.

\section{References}

1. WHO (2012) World Malaria Report 2012.

2. Day KP, Marsh K (1991) Naturally acquired immunity to Plasmodium falciparum Immunol Today 12: A68-71.

3. Gupta S, Snow RW, Donnelly CA, Marsh K, Newbold C (1999) Immunity to non-cerebral severe malaria is acquired after one or two infections. Nat Med 5: $340-343$.

4. Baird JK, Purnomo, Basri H, Bangs MJ, Andersen EM, et al. (1993) Age-specific prevalence of Plasmodium falciparum among six populations with limited histories of exposure to endemic malaria. Am J Trop Med Hyg 49: 707-719.

5. Wahlgren M, Björkman A, Perlmann H, Berzins K, Perlmann P (1986) AntiPlasmodium falciparum antibodies acquired by residents in a holoendemic area of Liberia during development of clinical immunity. Am J Trop Med Hyg 35: $22-29$

6. Langhorne J, Ndungu FM, Sponaas AM, Marsh K (2008) Immunity to malaria: more questions than answers. Nat Immunol 9: 725-732.

7. Drakeley CJ, Corran PH, Coleman PG, Tongren JE, McDonald SL, et al. (2005) Estimating medium- and long-term trends in malaria transmission by using serological markers of malaria exposure. Proc Natl Acad Sci U S A 102: 5108-5113.

8. Federal democratic republic of Ethiopia ministry of health $(\mathrm{FMoH})$ : National fiveyear strategic plan for malaria prevention and control in Ethiopia 2006-2010.

9. Amhara regional state Bureau of Agriculture (BoA) 2000 .

10. Population and housing census 2007, Population Census Commission, Federa democratic republic of Ethiopia.

11. Hodder AN, Crewther PE, Anders RF (2001) Specificity of the protective antibody response to apical membrane antigen 1. Infect Immun 69: 3286-3294.

12. Hoffmann EH, da Silveira LA, Tonhosolo R, Pereira FJ, Ribeiro WL, et al. (2001) Geographical patterns of allelic diversity in the Plasmodium falciparum malariavaccine candidate, merozoite surface protein-2. Ann Trop Med Parasitol 95: 117-132.

13. Theisen M, Soe S, Oeuvray C, Thomas AW, Vuust J, et al. (1998) The glutamate-rich protein (GLURP) of Plasmodium falciparum is a target for antibody-dependent monocyte-mediated inhibition of parasite growth in vitro. Infect Immun 66: 11-17

14. Iriemenama N, Khirelsiedc A, Nasra A, ElGhazali G, Giha HA, et al. (2009) Antibody responses to a panel of Plasmodium falciparum malaria blood-stage antigens in relation to clinical disease outcome in Sudan. Vaccine 27: 62-71.

15. Mamo H, Esen M, Ajua A, Theisen M, Mordmüller B, et al. (2013) Humoral immune response to Plasmodium falciparum vaccine candidate GMZ2 and its components in populations naturally exposed to seasonal malaria in Ethiopia. Malar J 12: 51.

16. Hogh B, Petersen E, Dziegiel M, David K, Hanson A, et al. (1992) Antibodies to a recombinant glutamate-rich Plasmodium falciparum protein: evidence for protection of individuals living in a holoendemic area of Liberia. Am J Trop Med Hyg 46: 307-313.

17. Dziegiel M, Rowe P, Bennett S, Allen SJ, Olerup O, et al. (1993) Immunoglobulin $\mathrm{M}$ and $\mathrm{G}$ antibody responses to Plasmodium falciparum glutamate-rich protein correlation with clinical immunity in Gambian children. Infect Immun 61: 103-108.

18. Pratt-Riccio L, Lima-Junior JC, Carvalho LJ, Theisen M, Espi'ndola-Mendes EC, et al. (2005) Antibody response profiles induced by Plasmodium falciparum glutamate-rich protein in naturally exposed individuals from a Brazilian area endemic for malaria. Am J Trop Med Hyg 73: 1096-1103.

19. de Stricker K, Vuust J, Jepsen S, Oeuvray C, Theisen M (2000) Conservation and heterogeneity of the glutamate-rich protein (GLURP) among field isolates and laboratory lines of Plasmodium falciparum. Mol Biochem Parasitol 111 123-130.

20. Chelimo K, Ofulla AV, Narum DL, Kazura JW, Lanar DE, et al. (2005) Antibodies to Plasmodium falciparum antigens vary by age and antigen in children in a malaria-holoendemic area of Kenya. Pediatr Infect Dis J 24: 680-684.

21. Thomas A, Trape J-F, Rogier C, Goncalves A, Rosario V, et al. (1994) High prevalence of natural antibodies against Plasmodium falciparum $83-\mathrm{kDa}$ apical membrane antigen (PF83/AMA-1) as detected by capture-enzyme-linked immunosorbent assay using full-length baculovirus recombinant PF83/AMA-I. Am J Trop Med Hvg 5l: 730-740.

22. Courtin D, Oesterholt M, Huismans H, Kusi K, Milet J, et al. (2009) The quantity and quality of African children's IgG responses to merozoite surface antigens reflect protection against Plasmodium falciparum malaria. PLoS One 4: e7590.

23. Wipasa J, Suphavilai C, Okell LC, Cook J, Corran PH, et al. (2010) Longlived antibody and B Cell memory responses to the human malaria parasites Plasmodium falciparum and Plasmodium vivax. PLoS Pathog 6: e1000770.

24. Richards JS, Beeson JG (2009) The future for blood-stage vaccines against malaria. Immunol Cell Biol 87: 377-390.

25. Taylor RR, Smith DB, Robinson VJ, McBride JS, Riley EM (1995) Human antibody response to Plasmodium falciparum merozoite surface protein 2 is serogroup specific and predominantly of the immunoglobulin G3 subclass. Infect Immun 63: 4382-4388.

26. Rzepczyk CM, Hale K, Woodroffe N, Bobogare A, Csurhes P, et al. (1997) Humoral immune responses of Solomon Islanders to the merozoite surface antigen 2 of Plasmodium falciparum show pronounced skewing towards antibodies of the immunoglobulin G3 subclass. Infect Immun 65: 1098-1100.

27. al-Yaman F, Genton B, Anders R, Taraika J, Ginny M, et al. (1995) Assessment of the role of the humoral response to Plasmodium falciparum MSP2 compared to RESA and SPf66 in protecting Papua New Guinean children from clinical malaria. Parasite Immunol 17: 493-501.

28. Aucan C, Traoré Y, Tall F, Nacro B, Traoré-Leroux T, et al. (2000) High immunoglobulin $\mathrm{G} 2$ ( $\mathrm{lgG} 2$ ) and low IgG4 levels are associated with human resistance to Plasmodium falciparum malaria. Infect Immun 68: 1252-1258.

29. Conway DJ, Machado RL, Singh B, Dessert P, Mikes ZS, et al. (2001) Extreme geographical fixation of variation in the Plasmodium falciparum gamete surface protein gene Pfs $48 / 45$ compared with microsatellite loci. Mol Biochem Parasito 115: 145-156.

30. Perraut R, Guillotte M, Drame I, Diouf B, Molez J, et al. (2002) Evaluation of anti-Plasmodium falciparum antibodies in Senegalese adults using different types of crude extracts from various strains of parasite. Microbes Infect 4: 31-35

31. Polley SD, Conway DJ, Cavanagh DR, McBride JS, Lowe BS, et al. (2006) High levels of serum antibodies to merozoite surface protein 2 of Plasmodium falciparum are associated with reduced risk of clinical malaria in coastal Kenya. Vaccine 24: 4233-4246

32. Polley SD, Tetteh KK, Lloyd JM, Akpogheneta OJ, Greenwood BM, et al. (2007) Plasmodium falciparum merozoite surface protein 3 is a target of allele-specific immunity and alleles are maintained by natural selection. J Infect Dis 195: 279-287.

33. Metzeger W, Okenu D, David R, Cavanagh D, Robinson J, et al. (2003) Serum $\mathrm{lgG} 3$ to the Plasmodium falciparum merozoite surface protein 2 is strongly associated with a reduced prospective risk of malaria. Parasite Immunol 25: 307-312.

34. Stanisic D, Richards J, McCallum F, Michon P, King C, et al. (2009) Immunoglobulin $\mathrm{G}$ subclass-specific responses against Plasmodium falciparum merozoite antigens are associated with control of parasitemia and protection from symptomatic illness. Infect Immun 77: 1165-1174.

35. Akpogheneta OJ, Duah NO, Tetteh KK, Dunyo S, Lanar DE, et al. (2008) Duration of naturally acquired antibody responses to blood-stage Plasmodium falciparum is age dependent and antigen specific. Infect Immun 76: 1748-1755.

36. Giha HA, Nasr A, Iriemenam NC, Balogun HA, Arnot D, et al. (2010) Agedependent association between IgG2 and IgG3 subclasses to Pf332-C231 antigen and protection from malaria, and induction of protective antibodies by sub-patent malaria infections, in Daraweesh. Vaccine 28: 1732-1739. 
Citation: Mamo H, Iriemenam NC, Berzins K, Petros B (2013) Antibodies by Non-Febrile, Smear-Negative Individuals from a Malaria Epidemic-Prone Setting in Ethiopia are Strongly Reactive to Plasmodium Falciparum Blood-Stage Vaccine Candidate Antigens. J Vaccines Vaccin 4: 200. doi: $10.4172 / 2157-7560.1000200$

Page 8 of 8

37. Jangpatarapongsa K, Sirichaisinthop J, Sattabongkot J, Cui L, Montgomery $\mathrm{SM}$, et al. (2006) Memory T cells protect against Plasmodium vivax infection. Microbes Infect 8: 680-686.

38. Elliott S, Payne P, Duffy M, Byrne T, Tham W, et al. (2007) Antibody recognition of heterologous variant surface antigens after a single Plasmodium falciparum infection in previously naïve adults. Am J Trop Med Hyg 76: 860-864.

39. Tongren JE, Drakeley CJ, McDonald SL, Reyburn HG, Manjurano A, et al. (2006) Target antigen, age, and duration of antigen exposure independently regulate immunoglobulin $\mathrm{G}$ subclass switching in malaria. Infect Immun 74 257-264.

40. Snow RW, Marsh K (2002) The consequences of reducing transmission of Plasmodium falciparum in Africa. Adv Parasitol 52: 235-264

41. Wahlgren M, Berzins K, Perlmann P, Persson M (1983) Characterization of the humoral immune response in Plasmodium falciparum malaria. II. IgG subclass levels of anti-P. falciparum antibodies in different sera. Clin Exp Immunol 54 $135-142$

42. Baird JK, Jones TR, Danudirgo EW, Annis BA, Bangs MJ, et al. (1991) Age- dependent acquired protection against Plasmodium falciparum in people having two years exposure to hyperendemic malaria. Am J Trop Med Hyg 45 65-76.

43. Riley E, Morris-Jones S, Blackman M, Greenwood B, Holder A (1993) A longitudinal study of naturally acquired cellular and humoral immune responses to merozoite surface protein (MSP) 1 of Plasmodium falciparum in an area of seasonal malaria transmission. Parasite Immunol 15:513-24.

44. Branch OH, Oloo AJ, Nahlen BL, Kaslow D, Lal AA (2000) Anti-merozoite surface protein-1 19-kDa IgG in mother-infant pairs naturally exposed to Plasmodium falciparum: subclass analysis with age, exposure to asexual parasitaemia, and protection against malaria. V. The Asembo Bay Cohort Project. J Infect Dis 181: 1746-1752.

45. Baird JK, Masbar S, Basri H, Tirtokusumo S, Subianto B, et al. (1998) Age-dependent susceptibility to severe disease with primary exposure to Plasmodium falciparum. J Infect Dis 178: 592-595.

46. Dondorp AM, Lee SJ, Faiz MA, Mishra S, Price R, et al. (2008) The relationship between age and the manifestations of and mortality associated with severe malaria. Clin Infect Dis 47: 151-157. 